\title{
A DVB-T Baseband Demodulator Design Based on Multimode Silicon IPs
}

\author{
Kai-Yuan Jheng, Tsung-Han Wu, Yi-Chiuan Wang, Jih-Chiang Yeo, Yu-Ju Cho, and An-Yeu Wu
}

Graduate Institute of Electronics Engineering, National Taiwan University, Taipei 10617, Taiwan, ROC

\begin{abstract}
In this paper, an implementation of a baseband demodulator for DVB-T systems is presented. We build several multimode SIPs and integrate them into a DVB-T baseband demodulator. The demodulator includes FFT, channel estimator, channel equalizer, deinterleavers, FECs, and descrambler. We utilize Simulink to establish a system-level simulation environment and use this system simulation model to evaluate performance. Moreover, the modules we used to integrate the demodulator are multimode SIPs so that the demodulator is able to meet the multimode feature of not only DVB-T (2K and $8 \mathrm{~K}$ modes) but DVB-H (4K mode) systems.
\end{abstract}

\section{INTRODUCTION}

Recent rapid progress in very large scale integrated (VLSI) circuit technology has led to an emerging theme "System-on-a-Chip" (SoC). With the increase in the density and complexity in VLSI circuit, the design costs for the development of a chip are also increased. It calls for rapid prototyping and design reuse of major silicon intellectual property (SIP) modules to alleviate the designer's effort and to speed up the design process. Therefore, we build several multimode SIPs and integrate them into terrestrial digital video broadcasting (DVB-T) systems [1]. Furthermore, the multimode SIPs also support the $4 \mathrm{~K}$ mode of handheld digital video broadcasting (DVB-H) systems [2].

In order to provide high data rate at extremely low bit error rate (BER) for MPEG-2 video data transmission, coded orthogonal frequency division multiplexing (OFDM) technology has been adopted in DVB-T systems. Two modes of operation, a $2 \mathrm{~K}$ mode and an $8 \mathrm{~K}$ mode, are defined for DVB-T and DVB-H transmissions. The $2 \mathrm{~K}$ mode is suitable for small single frequency networks (SFN). The $8 \mathrm{~K}$ mode can be used for small and large SFN. Exclusively for use in DVB-H systems, a third transmission mode the $4 \mathrm{~K}$ mode is defined. The $4 \mathrm{~K}$ mode aims to offer an additional trade-off between transmission cell size and mobile reception capabilities, providing an additional degree of flexibility for DVB-H systems. With the addition of a $4 \mathrm{~K}$ mode, DVB-H benefits from the compromise between the high speed small arcas SFN capability of 2K DVB-T and the lower speed but larger area SFN of $8 \mathrm{~K}$ DVB-T. In order to deal with various propagation conditions encountered in wireless broadcasting channel, there are many modes for DVB-T systems and we can choose an optimized combination through these parameters.

This work was supported by the National Science Council, Taiwan, ROC, under Grant NSC92-2215-E-002-020.

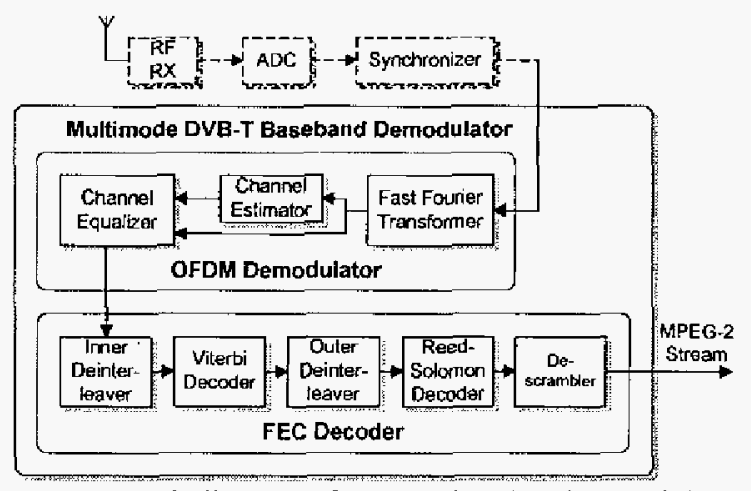

Fig. 1 Block diagram of DVB-T baseband demodulator

Table 1 Parameters of multimode DVB-T system

\begin{tabular}{|c|c|c|c|}
\hline Parameter & $2 \mathrm{~K}$ mode & $4 \mathrm{~K}$ mode & $8 \mathrm{~K}$ mode \\
\hline FFT Point & 2048 & 4096 & 8192 \\
\hline Camier Number & 1705 & 3409 & 6817 \\
\hline Useful Carrier Number & 1512 & 3024 & 6048 \\
\hline Guard Interval & $1 / 4 \cdot 1 / 8 \cdot 1 / 16 \cdot 1 / 32$ \\
\hline Code Rate & $1 / 2 \cdot 2 / 3 \cdot 3 / 4 \cdot 5 / 6 \cdot 7 / 8$ \\
\hline Modulation & QPSK $\cdot 16-\mathrm{QAM} \cdot 64-\mathrm{QAM}$ \\
\hline Useful Bitrate & $4.98-31.67 \mathrm{Mbps}$ \\
\hline
\end{tabular}

\section{SYSTEM OVERVIEW}

The block diagram of the DVB-T baseband demodulator is shown in Fig. 1. The received signal is first processed by the RF front-end and analog-to-digital converter (ADC). Then the digital signal is fed into the synchronizer to correct the frequency and timing offsets. The OFDM demodulator includes fast Fourier transformer (FFT), channel estimator and channel cqualizer. The inner decoder includes inner deinterleaver and Viterbi decoder with depuncture feature. The outer decoder is composed of $12 \times 17$ Forney convolutional deinterleaver and Reed-Solomon (RS) decoder. The channel bandwidth can be scaled to 8,7 , and $6 \mathrm{MHz}$ by adjusting the elementary period $\mathrm{T}$ to $7 / 64,1 / 8$, and $7 / 48$ is accordingly.

DVB-T system has various modes whose data rates are different. Their robustness against environment and channel is also different. Broadcasters can choose the most appropriate and efficient mode for broadcasting. The parameters of DVB-T systems for $8 \mathrm{MHz}$ channels are given in Table 1. The useful bitrate (UB) can be calculated by (1)

$$
\mathrm{UB}=\frac{1}{\mathrm{~T}} \times \frac{1}{1+\mathrm{Gl}} \times \frac{1512}{2048} \times \log _{2}(\mathrm{QAM}) \times \mathrm{CR} \times \frac{188}{204}
$$

where GI is the guard interval, QAM is the modulation and $\mathrm{CR}$ is the code rate of the inner decoder. 


\section{Simulation MOdeL}

The simulation model was written in the Matlab environment using Simulink. The system level model showed in Fig. 2 includes the modulator, Rayleigh channel (or Ricean channel), additive white Gaussian noise channel and the proposed multimode DVB-T baseband demodulator. The floating-point simulation is performed for functional verification and then fixed-point simulation is accomplished for hardware wordlength optimization. The parameters of channel can be obtained in the specification. [1].

There are many modes in DVB-T systems and some modes are more robust against environment while some are not. The required carrier-to-noise ratio (C/N) for quasi error free (QEF) reception of proposed demodulator in different modes are shown in Fig. 3. QEF means less than one uncorrected enror event per hour, corresponding to $B E R=10^{-11}$ at the input of the MPEG-2 demultiplexer. For QEF operation the associated inner BER is $2 \times 10^{-4}$. The total length of the bar is the required $\mathrm{C} / \mathrm{N}$ for QEF in the proposed demodulator and the lower part of the bar is the required $\mathrm{C} / \mathrm{N}$ for $\mathrm{QEF}$ under perfect channel estimation and without phase noise. The performance of the proposed demodulator only drops $2-3 \mathrm{~dB}$ in Ricean channel but needs improvement in Rayleigh channel for portable reception.

Through the fixed-point system simulation, the data and twiddle factor wordlength of the OFDM demodulator is specified as 14-bit and 10-bit respectively to obtain enough SNR under $40 \mathrm{~dB}$ AWGN channel. The wordlength and clock rate of each module is assigned as in Fig. 4.

\section{OFDM DEMODULATOR}

\subsection{Fast Fourier Transformer}

The FFT is one of the key modules in the implementation of OFDM systems. In conventional approach, computation is done by a single processor operated at a high clock rate. However, pipeline structure can operate at a lower clock frequency and is more preferable in low power application.

We adopt radix $-2^{2}$ FFT algorithm [3] which is a modified version of radix -4 FFT algorithm. A radix -4 butterfly can be divided into 2 radix- 2 butterflies. The twiddle factor between them can only be 1 or $-\mathrm{j}$. For hardware implementation, a complex multiplication for these twiddle factors can be

Fig. 2 DVB-T system simulation model in Simulink

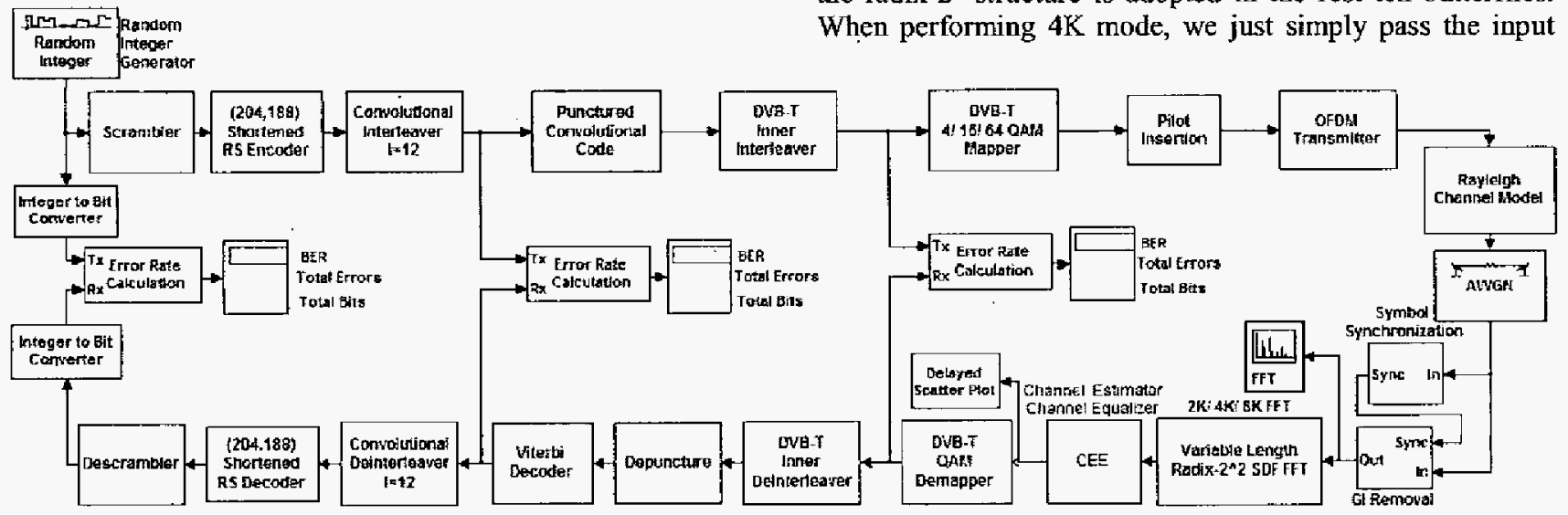

(a)

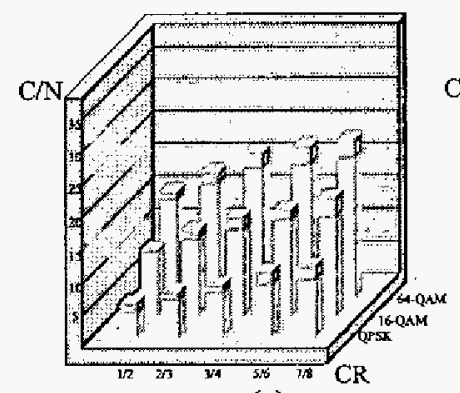

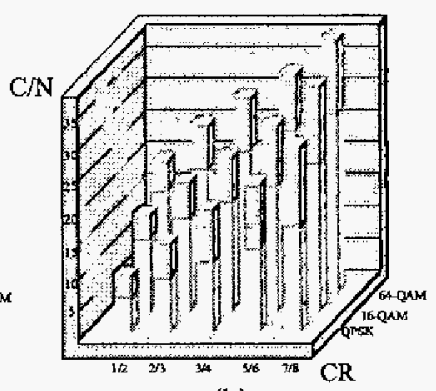

(b)
Fig. 3 The performance comparison of channel estimation under various modes between (a) Ricean channel and (b) Rayleigh channel.

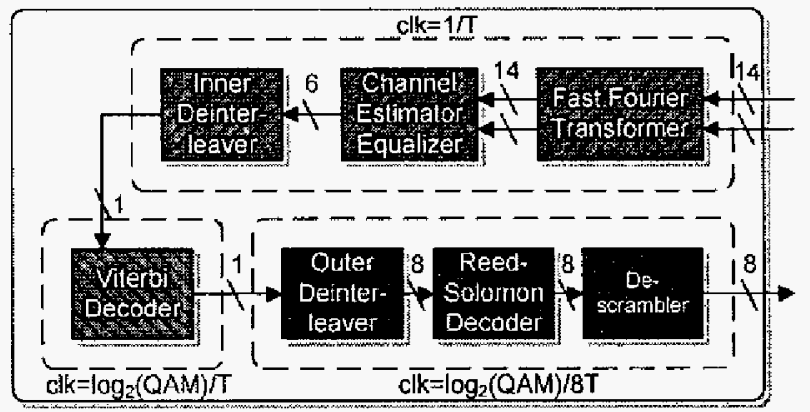

Fig. 4 The wordlength and clock rate of each module

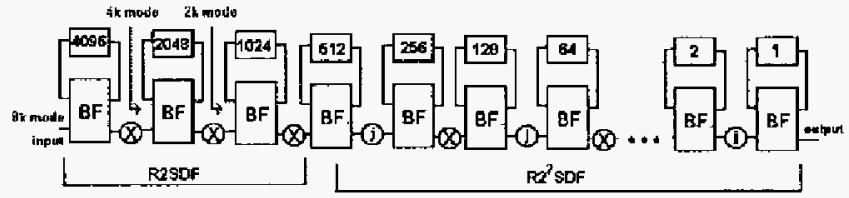

Fig. 5 Block diagram of the multimode $8 \mathrm{~K} / 4 \mathrm{~K} / 2 \mathrm{~K}$ FFT

realized by interchanging the real and imaginary part and sign inversion. Therefore Radix $-2^{2}$ FFT algorithm needs onty half of complex multipliers of radix-2 FFT and remains the simple control scheme as radix-2 FFT.

Multimode ( $2 \mathrm{~K}, 4 \mathrm{~K}$, and $8 \mathrm{~K}$ points) FFT computation can be realized by single delay feedback (SDF) architecture. Figure 5 shows a block diagram of the multimode pipeline FFT which is able to perform all $8 \mathrm{~K} / 4 \mathrm{~K} / 2 \mathrm{~K}$ operation. The radix -2 structure is still used in the first three butterflies and the radix $-2^{2}$ structure is adopted in the rest ten butterflies. When performing $4 \mathrm{~K}$ mode, we just simply pass the input 


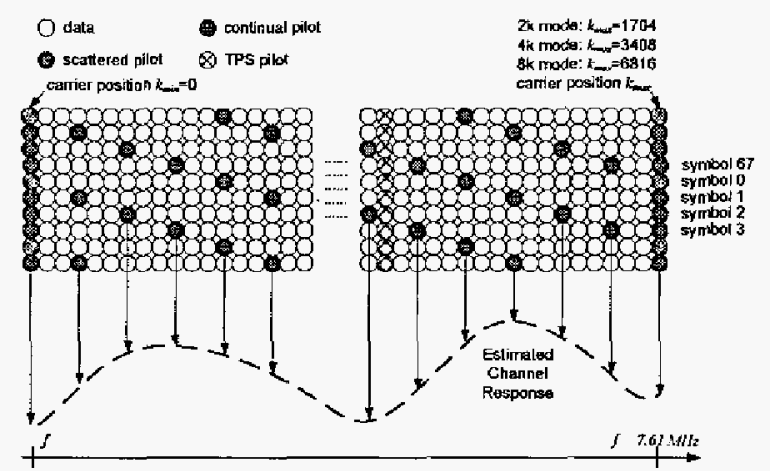

Fig. 6 The pilot pattern and ID linear interpolation method

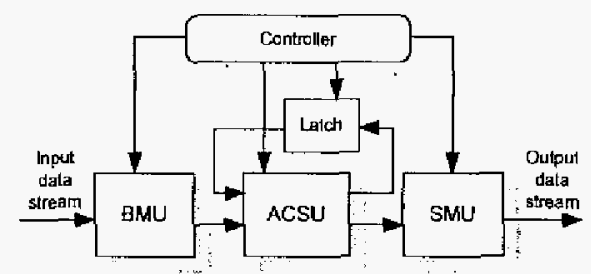

Fig. 7 Block diagram of Viterbi decoder

data to the $2^{\text {nd }}$ butterfly stage and skip the $1^{\text {st }}$ butterfly stage. The same, the input data are passed to the $3^{\text {rd }}$ stage and the $1^{\text {st }}$ and $2^{\text {nd }}$ stages are skipped in $2 \mathrm{~K}$ mode.

\subsection{Channel Estimator and Equalizer}

Channel estimation and equalization are also very important issues in OFDM systems. Immediate channel estimation and equalization methods must be performed for wireless multipath fast fading channel and mobile application. In addition to the transmitted data an OFDM frame contains scattered pilots, continual pilots, and transmission parameter signalling (TPS) carriers, which is shown in Fig. 6. For the symbol of index $n$ (ranging from 0 to 67), carriers for which index $k$ belongs to the subset

$\left\{k=K_{\min }+3 \times(n \bmod 4)+12 \mathrm{p} \mid \mathrm{p} \in Z, \mathrm{p} \geq 0, \mathrm{k} \in\left[\mathrm{K}_{\min } ; \mathrm{K}_{\max }\right]\right\}$ are scattered pilots. The scattered pilots have a period of four in time domain and twelve in frequency domain.

Considering efficient hardware implementation, we propose a simple 1D linear interpolation method for channel estimation. Let $\mathrm{R}(\mathrm{j}, \mathrm{k})$ denote the received $\mathrm{k}$-th scattered pilot of the $\mathrm{j}$-th symbol. In this method, scattered pilots from four successive OFDM symbols are first grouped together to obtain $\widetilde{\mathrm{R}}(j, \mathrm{k})$ which is decimated by three is shown in (2).

$$
\widetilde{R}(j, \vec{k})=\sum_{i-j-3}^{L} R(i \bmod 68, k)
$$

Then the estimated received scattered pilot carriers $\hat{R}(j, k)$ can be obtained from interpolating $\widetilde{R}$ as in (3).

$$
\hat{\mathrm{R}}(\mathrm{j}, \mathrm{k})=\left(1-\frac{\mathrm{k} \text { mod } 3}{3}\right) \widetilde{\mathrm{R}}\left(\mathrm{j}, 3\left(\frac{\mathrm{k}}{3}\right)\right)+\frac{\mathrm{k} \text { mod } 3}{3} \tilde{\mathrm{R}}\left(\mathrm{j}, 3\left\lfloor\frac{\mathrm{k}}{3}\right\rfloor+3\right)
$$

where $\lfloor x\rfloor$ denotes the interger part of $x$. Then $\widetilde{R}$ is correspondingly divided by the transmitted "boosted" power level $\pm \frac{4}{3}[1]$ to evaluate the estimated channel gain $\hat{\imath}$.

$$
\hat{H}(j, k)=\left(I-\frac{k \operatorname{mad} 3}{4}\right) \widetilde{R}\left(1,3\left\lfloor\frac{k}{3}\right\rfloor\right)+\frac{k \bmod 3}{4} \tilde{R}\left(1,3\left\lfloor\frac{k}{3}\right\rfloor+3\right)
$$

Thus, using the proposed ID linear interpolation method, the estimated channel gain can be obtained casily by shift-and add operation. Then a complex divider is used as a channel equalizer. The multimode channel estimation and equalization module can also work at $2 \mathrm{~K}, 4 \mathrm{~K}$, and $8 \mathrm{~K}$ modes.

\section{FEC DECODER}

\subsection{Inner Deinterleaver}

Inner interleaving consists of a bit and a symbol interleaver. The interleaving process results in frequency interleaving instead of time interleaving.

\subsubsection{Symbol Deinterleaver}

The symbol deinterleaver is a block based deinterleaver. It acts on blocks of 1512 ( $2 \mathrm{k}$ modc), 3024 (4k modc), or 6048 ( $8 \mathrm{k}$ mode) v-bit words, where $\mathrm{v}$ is the number of bits per modulation symbol. The data reordering can be implemented as memory to memory transfer. Data from a memory can be moved to a different address in another memory [4]. The v-bit words $\mathrm{y}^{\text {in }}=\left[\mathrm{y}_{0}, \mathrm{y}_{1}, \ldots, \mathrm{y}_{\mathrm{v}-1}\right]$ are read sequentially into a vector $Y^{\text {in }}=\left(y_{0}^{\text {in }} y_{1} y_{1}^{\text {in }}, \ldots, y_{\text {Ninax-1 }}^{\text {in }}\right)$. The deinterleaved vector $Y^{\text {out }}=$ $\left(y^{\text {out }}, y^{\text {nut }}, \ldots, y^{\text {out }}{ }_{\text {Nmax }-1}\right)$ is defined by:

$$
y^{\text {out }}=y^{\text {in }}{ }_{11} \text { (19) } \quad \text { for even OFDM symbols }
$$

$\mathrm{y}^{\text {out }}{ }_{\mathrm{H}(\mathrm{q})}=\mathrm{y}_{\mathrm{q}}^{\mathrm{in}} \quad$ for odd OFDM symbols where $q-0,1, \ldots, N_{\max }-1$ and $\mathrm{N}_{\max }=1512$ in $2 \mathrm{k}$ mode, $\mathrm{N}_{\max }=$ 3024 in $4 \mathrm{k}$ mode, and $\mathrm{N}_{\max }=6048$ in $8 \mathrm{k}$ mode. $\mathrm{H}(\mathrm{q})$ is the permutation function for symbol interleaving [1].

\subsubsection{Bit Deinterleaver}

The bit deinterleavers are also block based and the block size is 126 bits. Each block is cyclically shifted for a different bit position and then outputs [1][4]. When operating at different types of modulation (QPSK, 16-QAM or 64-QAM), there are 2, 4 or 6 sub-data output streams. We just enable the corresponding memory banks in bit deinterleaver which are multimode for different modulation. 'The sub-data streams are then combined and sent to Viterbi decoder..

\subsection{Viterbi Decoder}

A depuncturing process before decoding the original rate-1/2 code based on Viterbi algorithm is necessary. In depuncturing process, we insert some dummy data at the positions that have been deleted in transmitter according to the perforation pattern; therefore the multimode Viterbi decoder can deal with the following code rate: $1 / 2,2 / 3,3 / 4$, $5 / 6$ and $7 / 8$.

Figure 7 is the block diagram of a Viterbi decoder which is mainly divided into branch metric calculation unit (BMU), add-compare-select unit (ACSU) and survivor memory unit (SMU) [5]. BMU performs the computation of each branch metric for every input data. The computation is achieved by using the Hamming distance definition. ACSU computes the accumulative sum of each branch metric for current state and selects the one with minimum hamming distance to replace the current state metric. Then a certain number of recursion should be performed and the most-likely path would be chose for decoding. Therefore, a SMU is necessary to store the metric of each state and the trellis at each recursion. After one survivor depth cycle, we shall trace back from the contents of survivor memory and output the decoded data. 


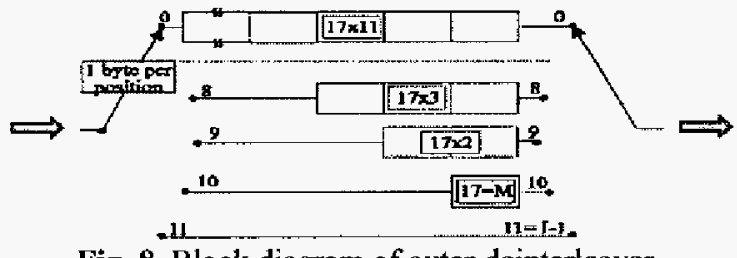

Fig. 8 Block diagram of outer deinterleaver

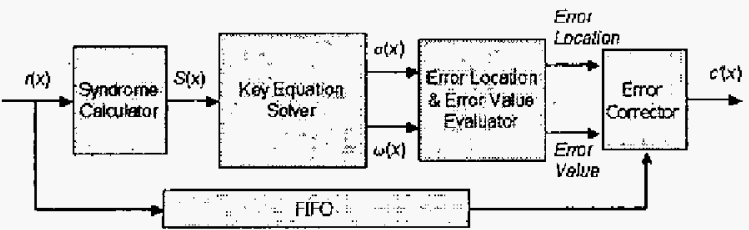

Fig. 9 Block diagram of RS deconder

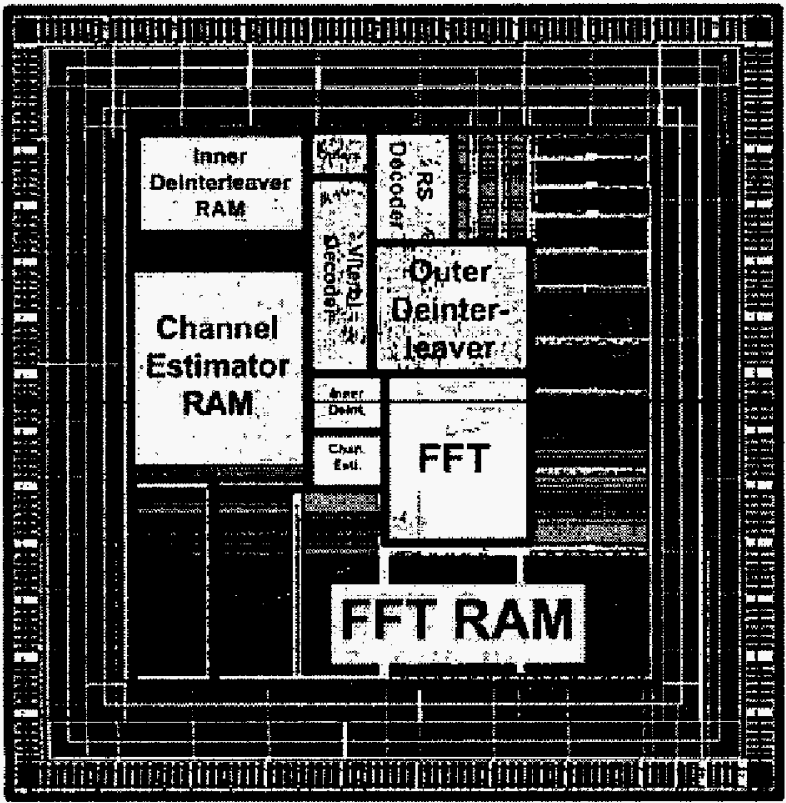

Fig. 10 The layout of the multimode DVB-T baseband demodulator

Table 2 The summary of the prototypical design

\begin{tabular}{|c|c|}
\hline Technology & UMC 0.18 $\mu \mathrm{m}$ CMOS 1P6M \\
\hline Gate Count & $1,394,904$ (SRAM 76\%) \\
\hline Core Size & $3.9 \mathrm{~mm} \times 4.0 \mathrm{~mm}$ \\
\hline Working Voltage & $1.8 \mathrm{~V}$ \\
\hline Nóminal Frequency & $54.86 \mathrm{MHz}$ \\
\hline Power Consumption & $307 \mathrm{~mW} @ 31.67 \mathrm{Mbps}$ \\
\hline
\end{tabular}

\subsection{Outer Deinterleaver}

The combination of RS code with interleaving which spreads out burst errors in time domain is effective to correcting burst errors caused by impulse noise in the channel.

In this system, the encoded symbols are sequentially shifted into a register bank with 12 branches, and each branch includes 17 bytes. Similarity, the symbols are shifted out from the register bank in received side. Figure 8 shows the structure of outer deinterleaver.

\subsection{Reed-Solomon Decoder}

In this system, RS $(204,188)$ shortened code, derived from the original systematic $\mathrm{RS}(255,239)$ code, shall be applied to each transport packet (188 bytes). The shortened RS code may be implemented by adding 51 bytes, all set to zero, before the information bytes at the input of an $\operatorname{RS}(255,239)$ encoder. After the RS coding procedure these null bytes shall be discarded, lcading to a $\mathrm{RS}$ code word of $\mathrm{N}=204$ bytes.

RS decoding process [6] can be mainly divided into three parts, syndrome calculation, solving key equation and error calculation. The block diagram of RS decoder is showed in Fig. 9.

\section{IMPLEMENTATION}

The multimode DVB-T baseband demodulator is realized by. Verilog code and functionally verified by system simulation. We utilize the Synopsys Design Compiler to synthesize the RTL codes using UMC Artisan $0.18 \mu \mathrm{m}$ standard cell library. And then Apollo is used to place and route the prototypical layout as shown in Fig. 10. A brief summary of the prototypical design is given in Table 2 .

\section{ConcLusions}

A DVB-T baseband demodulator design based on multimode SIPs is presented. We utilize Simulink to establish a system-level simulation environment. Then we use this system simulation model to evaluate performance. Our demodulator, using the proposed simple $1 \mathrm{D}$ linear interpolation method for channel estimation, well performed in Ricean channel. However, we need to figure out some solution to improve the performance in Rayleigh channel. The modules we used to integrate the demodulator are multimode SIPs so that the demodulator is able to meet the multimode feature of not only DVB-T (2K and $8 \mathrm{~K}$ modes) but DVB-H ( $4 \mathrm{~K}$ mode) systems.

\section{REFERENCES}

[1] European Telecommunications Standards Institute (ETSD) "Digital video broadcasting (DVB); Framing structure, channel coding, and modulation for digital terrestrial television," ETSI EN 300744 v1.5.1, Jun. 2004.

[2] ETSI, "Digital video broadcasting (DVB); Transmission system for handheld terminals (DVB-H)," ETSI EN 302304 v1.1.1, Jun. 2004.

[3] S. He and M. Torkelson, "A new approach to pipeline FFT processor," IEEE Int. Parallel Processing Symp., pp. 766-770, Apr. 1996.

[4] L. Horvath, et al., "A novel, high-speed, reconfigurnble demapper-symbol deinterleaver architecture for DVB-T," IEEE Int. Symp. Circuits Syst., pp. 382-385, May 1999.

[5] H.-L. Lou, "Implementing the Viterbi algorithm," IEEE Signal Processing Magazine, pp. 42-52, Sep. 1995.

[6] J.-C. Yeo, H.-Y. Hsu, and A.-Y. Wu, "A scalable Reed-Solomon decoding processor based on unified finite-field processing element design," to appear, IEEE Workshop Signal Processing Systems (SiPS), Oct. 2004. 